\title{
SURTOS DE SALMONELOSE (Salmonella typhimurium) EM Cavia porcellus
}

\author{
SALMONELLOSIS (Salmonella typhimurium) OUTBREAKS IN Cavia porcellus
}

Selene Dall'Acqua COUTINHO'; I.uzinete Alves da SII,VA²; ldércio Luiz SINHORINI'; Vânia Maria de (ARVAI.HO';

Elizabeth Oliveira da COSTA*

\begin{abstract}
RESUMO
Relatam-se dois surtos de salmonelose em cobaias (C'avia porcellus), envolvendo um total de 70 animais mantidos em biotério, que manifestaram emaciação progressiva, prostração. hipertemia, pelos arrepiados e rarefeitos. Após o início dos sintomas, 48,57\% dos animais (34/ 70) evoluíram para a morte, sendo os demais sacrificados com o intuito de se controlar os surtos. Na necrópsia. coltheram-se fragmentos de fígado, haço, rins, pulmões e intestinos para exames histopatológico e microbiológico. Ao exame microbiológico obteve-se o isolamento de cepas caracterizadas bioquímica e sorológicamente como Salmonella typhimurium. () exame histopatológico dos fragmentos de orgãos corados pela hematoxilina-eosina revelou a presença de lesões condizentes com quadro septicêmico por este microrganismo. O presente trabalho alerta quanto ao risco existente na utilizacão de animais de experimentaçāo portadores dessa bactéria, já que poderiam comprometer experimentos por uma possível interferência nos resultados, além de representarem fonte de inlecção em biotérios e laboratórios.
\end{abstract}

UNITERMOS: Salmonelose; Salmonella typhimurium; Cobaias; Zoonoses; Biotério

\section{INTRODUÇÃO}

A salmonelose constitui importante zoonose que tem afetado com frequiência diferentes espécies de animais de laboratório. ${ }^{8.9}$.

Apesar de poucos trabalhos abordarem este problema $\mathrm{em}$ nosso pais, sabe-se da ocorrência dessa infecção em animais de experimentação, como relatado em coelhos por FARI$\mathrm{NHA}^{3}$ (1980).

Em cobaias (Cavia porcellus), vários surtos de salmonelose êm sido descritos em outros países.7.10; entretanto, no 3rasil, a doença nesta espécie animal não tem sido notificada, alvez pela falta de diagnóstico, ou mesmo, de publicação dos ıchados eventuais.

Jo presente relato descrevem-se dois surtos de samonelose $\mathrm{m}$ cobaias de mesma procedência, caracterizados por alta axa de letalidade, as quais seriam utilizadas em trabalho de esquisa.

\section{MATERIAL E MÉTODO}

Is dois surtos ocorridos em épocas sazonais diferentes nverno e verão), demonstraram características similares.

Pós-Graduanda - Instituto de Ciências Biomédicas da USP

Professor Assistente Doutor - Instituto de Ciências Biomédicas da USP

Professor Assistente Doutor - Faculdade de Medicina Veterinária e Zootecnia da USP

Professor Associado - Faculdack de Medicina Veterinária e Zoxtecnia da USP
Setenta (70) cobaias ( 37 no inverno e 33 no verão) de ambos os sexos, adultas ( $350-400 \mathrm{~g}$ ), aparentemente sadias, apresentaram hipertermia (temperatura retal $>40^{\prime \prime} \mathrm{C}$ ), emaciação progressiva, prostração e pelos arrepiados e rarefeitos. A evolução dá doença levou 34 animais à morte 10 a 20 dias após o início dos sintomas. Realizou-se necrópsia das cobaias, colhendo-se fragmentos de fígado, baço, rins, pulmões e intestinos para exame histopatológico, sendo fixados $\mathrm{cm}$ líquido de Bouin e emblocados em parafina. Foram realizados cortes de $5 \mu \mathrm{m}$ de espessura, corados pelo método da Hematoxilina-Eosina (HE).

Colheram-se ainda, assepticamente, fragmentos dos órgãos referidos para a realização do exame microbiológico, empregando-se como meio de cultivo caldo e ágar infusão de cérebro-coração*, incubados a $37^{\circ} \mathrm{C}$ por 24 horas, após a sua inoculação. Procedeu-se, a seguir, o isolamento de colônias, as quais foram identificadas bioquímica ${ }^{2}$ e sorologicamente**. As cepas isoladas foram também submetidas a testes de sensibilidade a antimicrobianos***, empregando-se a técnica de Kirby \& Bauer'.

\section{RESULTADOS}

Macroscopicamente a necrópsia dos animais revelou a presença de lesōes nos pulmões, que se apresentaram com

* BHI-Difco

- Identificação sorológica realizada no Instituto Adolpho Lutz de Săo Paulo

*** Multidiscos Laborclin 
(OUTINHO.S.D.A.: SILVA.L.A.: SINHORINI, I.L.: ('ARVALIIO, V.M.: ('OSTA. E.O. Surtos de sulınonelos" (Salmonella typhimurium) em (avia porcellus. Hraz. J. vet. Res. anim. Sici., Sáo P'uulo, v. 31, n.3/4, p. 233-37, 1994

alterações de coloração e múltiplas áreas de aspecto esbranquiçado ao conte. O fígado das cobaias demonstrou aumento de volume e consistência friável. Ao corte, notou-se a presença de nódulos esbranquiçados contendo material purulento, sinais estes que também foram observados no baço. Nos animais em que ocorreram alterações no trato

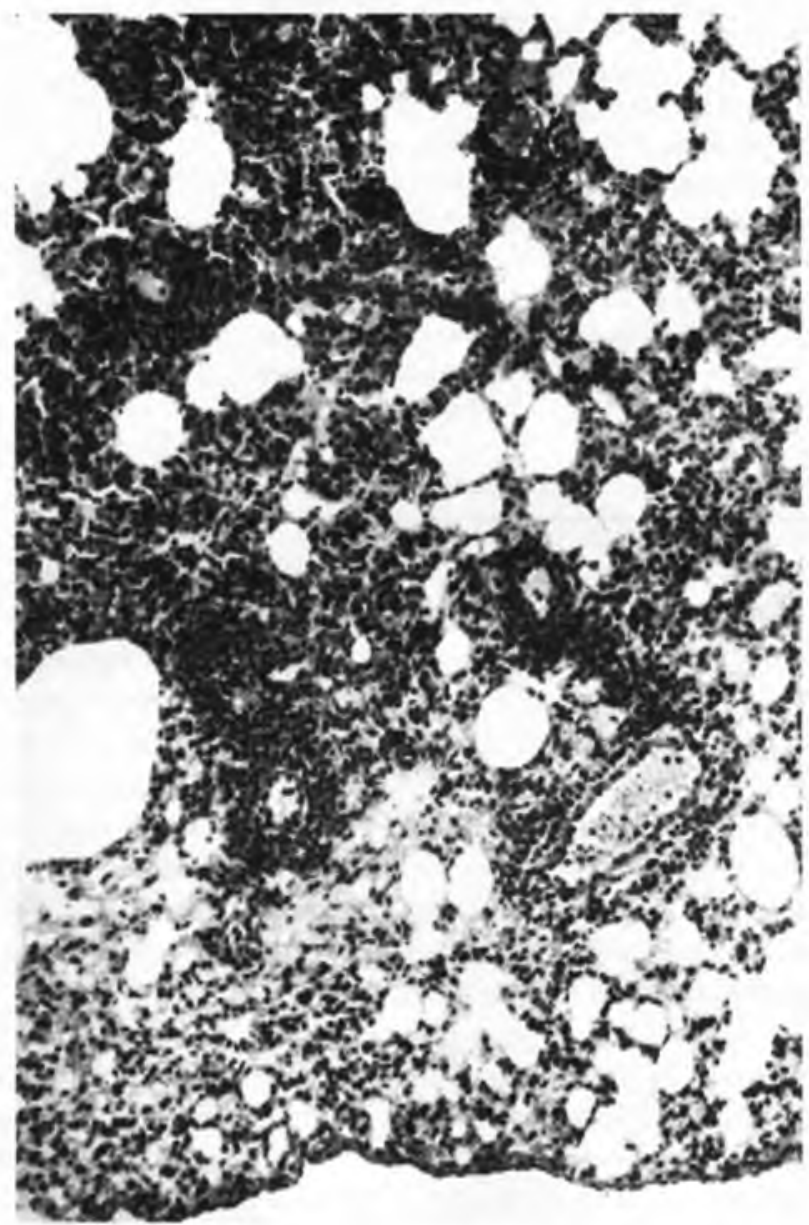

\section{FIGURA 2}

Corte histológico de fígado de cobaia corado pelo método da hematoxilina-eosina. Notar a presença de massa necrótica (N), circundada por infiltrado inflamatório composto predominantemente por células polimorfonucleares (PN) e por mononucleares ( $\mathrm{MN}$ ), e esboço capsular de tecido conjuntivo (TC). $165 x$. gastro-entérico, essas consistiram de processos congestivohemorrágicos.

Histologicamente as seguintes alterações foram observadas no exame dos órgãos acima citados: pneumonia intersticial crônica (Fig. 1), hepatite purulenta focal (Fig. 2), glomerulonefrite crônica e hemorragia intersticial (Fig. 3), histiocitose intrafolicular (Fig. 4) e enterite necrótica (Fig. 5).

\section{FIC;URA I}

Corte histológico de pulmão de cobaia corado pelo método da hematoxilina-eosina. Notar intenso infiltrado de células mononucleares no interstício. $165 x$.

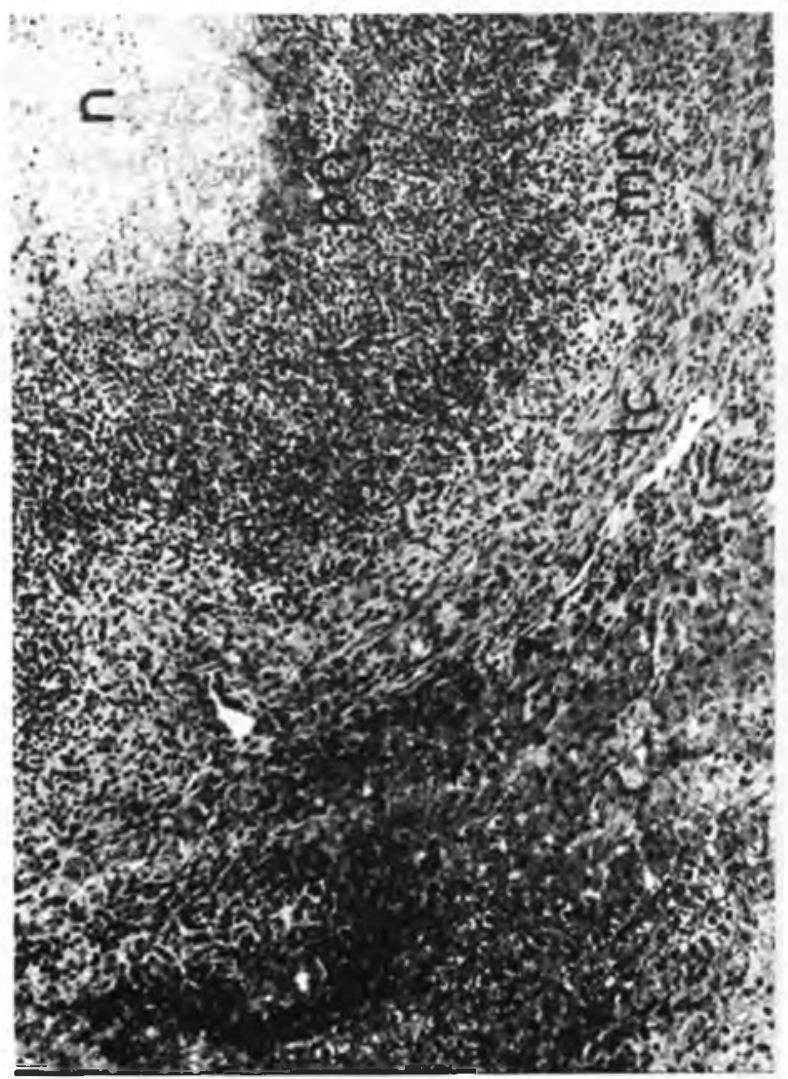




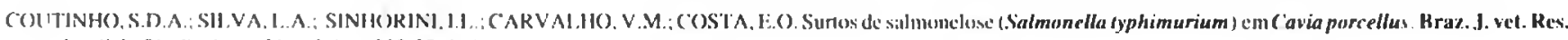

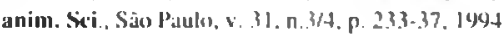

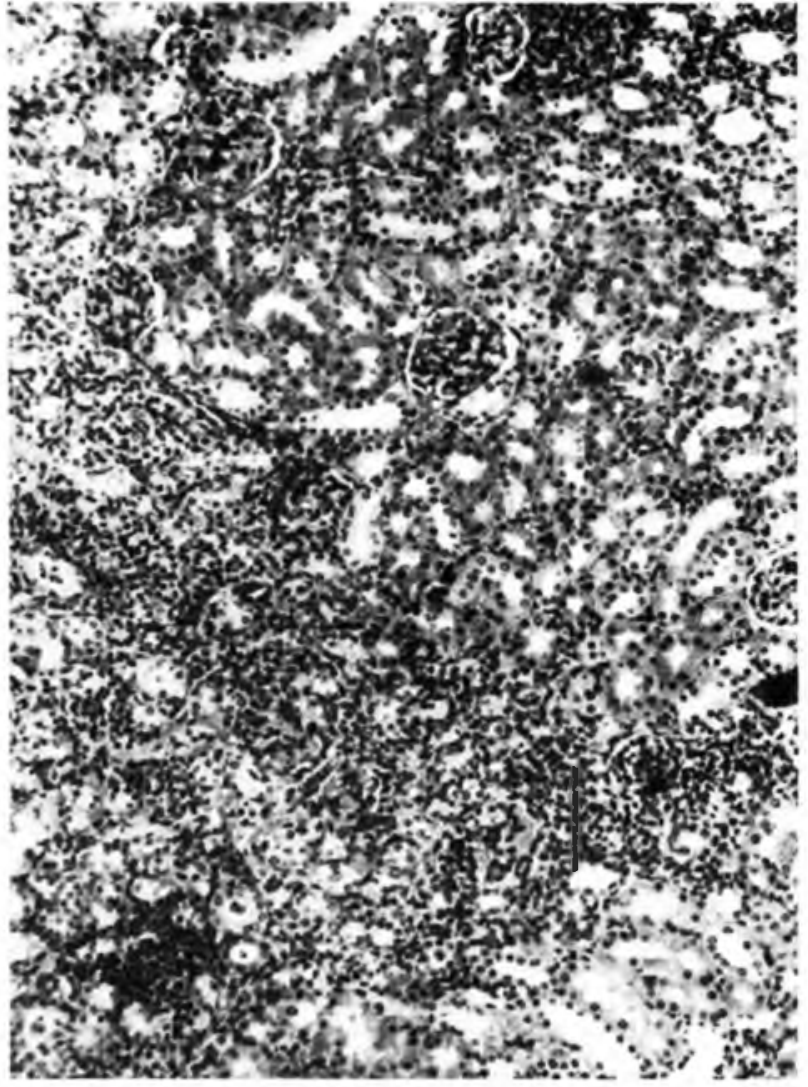

FIC;URA 4

Corte histológico de baço de cobaia corado pelo método da hematoxilina-eosina. Notar a presença de histiocitose intrafolicular. $16.5 x$.

\section{FIGURA 3}

Corte histológico de rim de cobaia corado pelo método da hematoxilina-cosina. Notar infiltrado de células mononucleares: comprometendo glomérulos e interstício. $16.5 \mathrm{x}$.

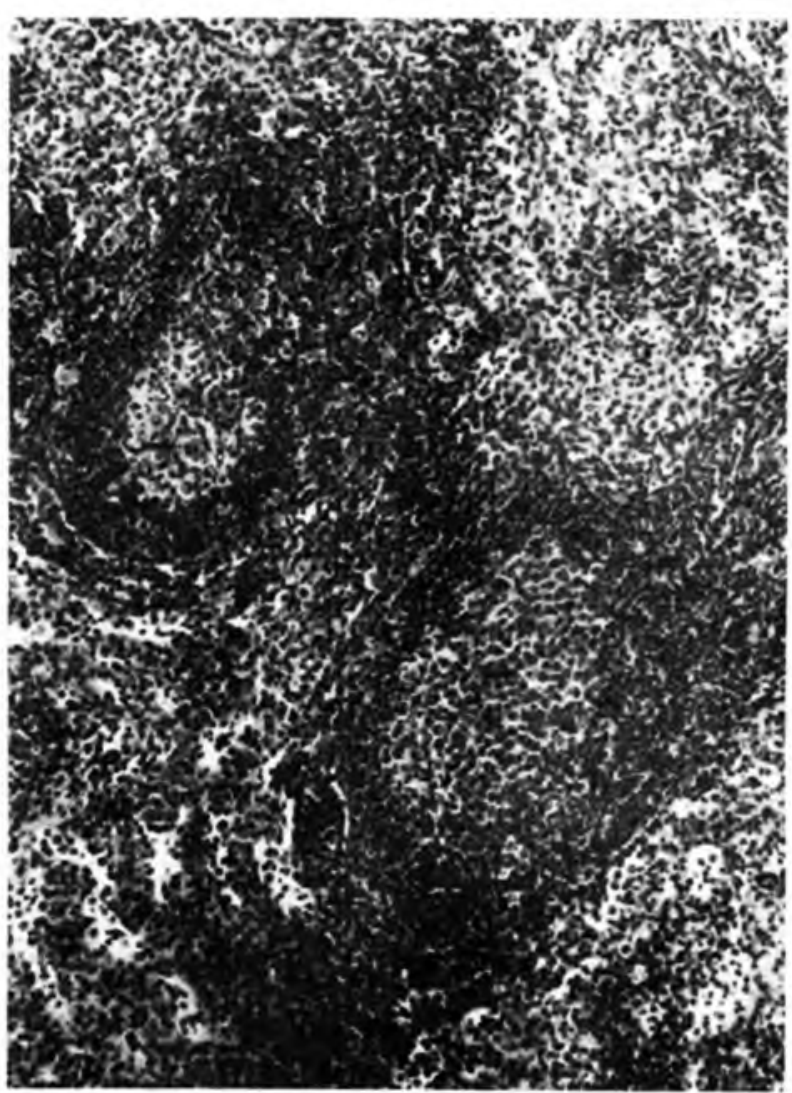


(OUTINIJO.S.D.A.: SILVA. L.A.; SINHORINI, I.L.; CARVAI.HO, V.M.; COSTA. F.O. Surtos de salmoneluse (Salmonella typhimurium) cm Cavia porcellus. Hraz. J. vet. Res. anim. Sci.. Săo Paulo, v. 31, n.3/4, p. 233-37. 1994.

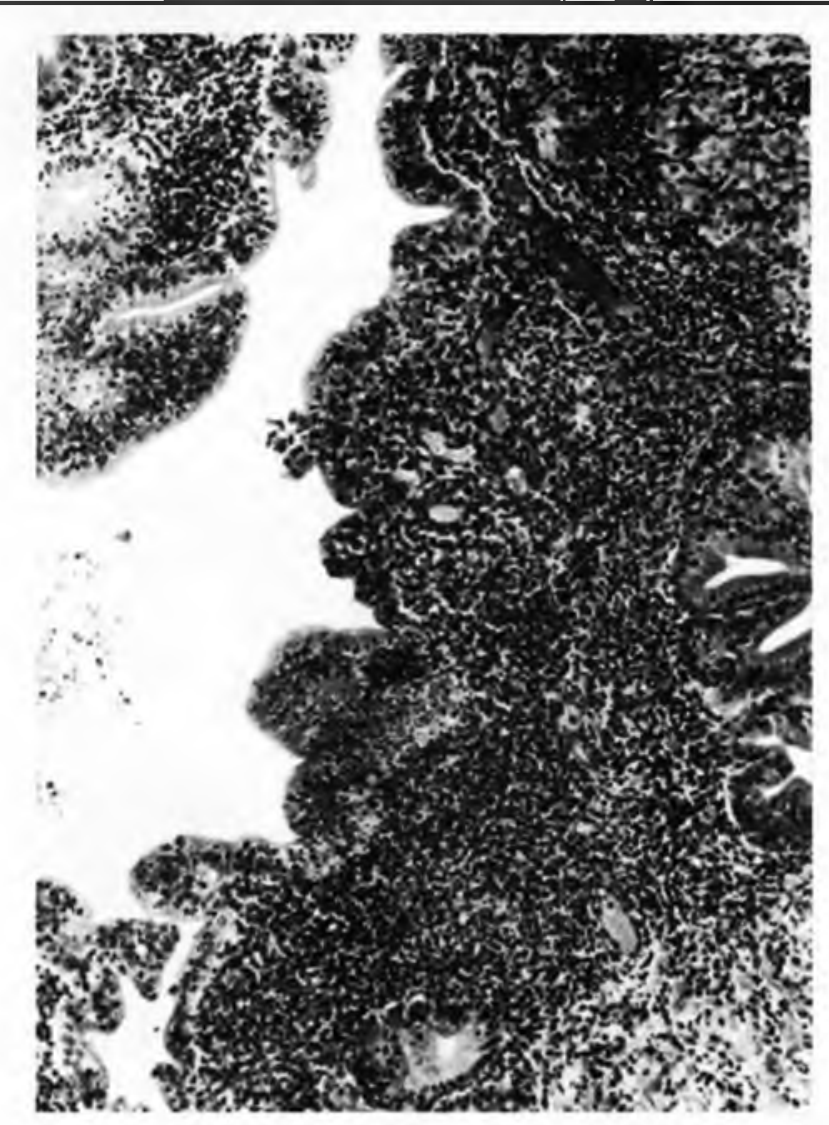

FIGURA 5

Corte histológico do intestino de cobaia corado pelo método da hematoxilina-eosina. Notar a presença de necrose das vilosidades, que se apresentam infiltradas por células polimorfonucleares e mononucleares. $165 \mathrm{x}$.

\section{Quadro 1}

Comportamentodas amostras deSalmonellatyphimurium frente a antimicrobianos.

\begin{tabular}{|cl|c|l|}
\hline$*$ & Gentamicina & $(10 \mu \mathrm{g})$ & Sensível \\
\hline$*$ & Amicacina & $(30 \mu \mathrm{g})$ & Sensível \\
\hline$*$ & Ampicilina & $(10 \mu \mathrm{g})$ & Sensível \\
\hline$*$ & Cefoxitina & $(30 \mu \mathrm{g})$ & Sensível \\
\hline$*$ & Cloranfenicol & $(30 \mu \mathrm{g})$ & Sensível \\
\hline$*$ & Tobramicina & $(10 \mu \mathrm{g})$ & Sensível \\
\hline$*$ & Sulfazotrim & $(25 \mu \mathrm{g})$ & Sensível \\
\hline$*$ & Carbenicilina & $(100 \mu \mathrm{g})$ & Intermediário \\
\hline$*$ & Netilmicina & $(30 \mu \mathrm{g})$ & Intermediário \\
\hline$*$ & Cefalotina & $(30 \mu \mathrm{g})$ & Intermediário \\
\hline$*$ & Tetraciclina & $(30 \mu \mathrm{g})$ & Resistente \\
\hline$*$ & Polimixina B & $(300 \mu \mathrm{g})$ & Resistente \\
\hline
\end{tabular}

No exame microbiológico dos fragmentos de órgãos obtevese, em 9 dos animais necropsiados, o isolamento deSalmonella typhimurium, cujo comportamento frente aos antimicrobianos testados é mostrado no Quad. 1.

\section{DISCUSSÃO}

Muitas são as afeç̧ões que acometem as diferentes espécies de animais de laboratório, destacando-se a salmonelose.

Em cobaias, a ocorrência de determinados sorotipos de Salmonella $\mathrm{sp}$, principalmente S. typhimurium, tem sido referida por vários autores ${ }^{6,9,10}$, sendo os resultados obtidos nos dois surtos estudados concordantes com os encontrados na literatura consultada.

Neste relato os animais analisados, procedentes de uma mesma colônia, manifestaram, cerca de 20 dias após o seu transporte ao novo biotério, sintomas semelhantes aos descritos por outros autores em surtos de salmonelose $\mathrm{em} \mathrm{C}$. porcellus $^{7.10}$. Como as cobaias apresentavam-se aparentemente sadias ao saírem do criatório, acredita-se que o desenvolvimento da salmonelose-clínica tenha ocorrido em animais, possivelmente, portadores do microrganismo, devido ao "stress" de transporte e adaptação ao novo local, já que neste não existiam problemas dessa natureza.

$O$ aspecto de disseminação do processo infeccioso por toda economia animal, revelado pelos exames microbiológico $\mathrm{e}$ histopatológico, demonstra quadro septicêmico, compatível com infecção por Salmonella $\mathrm{sp}^{10}$.

A taxa de letalidade foi de $48,57 \%$ (34/70), sendo os sobreviventes sacrificados, como método de controlc dos surtos, a fim de impedir a disseminação do microrganismo por animais eventualmente portadores. Cabe ressaltar que, a partir dos fragmentos de órgãos das cobaias sacrificadas, também se obteve o isolamento de Salmonella typhimurium.

Além de possível interferência no andamento e resultados dos experimentos que utilizam esses animais, a ocorrência do estado de portador de Salmonella sp em animais de laboratório apresenta-se como problema de saúde pública. devido ao sério risco de infecção para as pessoas que os manuseiam. A possibilidade de infecção humana no biotério motivou a realização de testes de sensibilidade com as cepas isoladas, pois o resultado obtido no antibiograma seria o suporte de uma terapia inicial.

Uma vez que esta infecção pode apresentar-se nos criatórios sob a forma de salmonelose-infecção, nem sempre detectável clinicamente $^{4}$, os resultados apresentados nesta investigação alertam aos que trabalham com animais de laboratório, particularmente C. porcellus, sobre a existência do problema em nosso país. 
COUTINHO, S.I) A.; SII.VA, 1. A.; SINHORINI. 1.L.; CARVALhO, V.M.; COSTA, F..O. Surtos de salmoneloxe (Salmonella typhimurium) em Cavia porcellus. Bryz. J. vel. Res. anim. Sci.. São Paulo. v. 31, n.3/4, p. 233-37. 1994.

\section{AGRADECIMENTO}

À médica veterinária Maria Christina Christovão Ramos, pelo auxílio prestado na execução deste trabalho.

\section{SUMMARY}

Two outbreaks of salmonellosis in guinea pigs (Cavia porcellus), are referred in a total of 70 animals maintained in animal facility, that showed loss of weigh, dullness, high body temperature and rough dull hair coat. Death occurred in $48.57 \%$ ( 34 / 70) a few days after showing the clinical signs. Fragments of liver, spleen, kidneys, lungs and intestines were collected during necropsy. The isolated colonies were characterized biochemically and serologically as Salmonella typhimurium. The histopathological lindings in the fragments stained by hematoxilin-eosin agreed with septicaemic condition. The present investigation shows the risk of carrier animals used in experimental trials to interfere with the obtained results; besides those animals represent a source of infection of this zoonosis in animal facilities and laboratories.

UNITERMS: Salmonellosis: Salmonella typhimurium; Guinea pigs; 7oonoses: Vivarium

\section{RFFERÊNCIAS BIBLIOGRÁFICAS}

()1-BAUER, A.W.; KIRBY, W.M.M.; SHERRIS, J.C.; TURCK, M. Antibodic susceptibility testing by a standardized single disk method. American Journal of Clinical Pathology, v.45, p.493-6, 1966.

(02-EDWARDS, P.R.; EWING, W.H. Identification of Enterobacteriaceae. 3.ed. Minneapolis, Burgess Publishing, 1972.

03-FARINHA, S.B. Surto de salmonelose em coelhos domésticos. Revista Latino Americana de Cunicultura. v. I, p.53-6, 1980.

()4-GIORGI, W. Animais domésticos como portadores de salmonelas: significado epidemiológico e sua relação com a saúde pública. Atualidades Veterinárias, v.3, p.47, 1974.

05-JA Y ASHEELA, M.; GOWAL, K.N.; JOHN, P.C.; MAGO, M.L.; SAXENA, S.N. An outbreak of salmonellosis in guinea-pigs. Indian Veterinary Journal, v.3, p. 100912, 1985.

06-JOHN, P.C.; GOWAL, K.N.; JAYASHEELA, M.; SAXENAS, N. Natural course of salmonellosis in a guinea pigs colony. Indian Veterinary Journal, v.65, p.200-4, 1988.
07-ONYEKABA, C.O. Clinical samonellosis in a guinea pig colony caused by a new Salmonella serotype, Salmonella ochiogu. Laboratory Animals, v.17, p.2136, 1983.

08-RAO, M.R.K.; CHAR, N.L. Enterobacteria isolated from pathological conditions of laboratory animals. Indian Veterinary Journal, v.63, p.179-82, 1986.

09-RAY, J.P.; MALLICK, B.B. Public health significance of Salmonella infections in laboratory animals. Indian Veterinary Journal, v.47, p.1033-7, 1970.

10-SRIVASTAVA, N.C.; KHERA, S.S. Salmoncllosis in guinea pigs. Indian Veterinary Journal, v.58, p.9212,1981 .
Recebido para publicacão em 26/03/93 Aprovado para publicacão em 04/02/94 